\title{
CONTROL DE Euphorbia dentata CON DIFERENTES FORMULACIONES DE GLIFOSATO EN SOJA TRANSGENICA RESISTENTE A GLIFOSATO
}

\author{
Víctor F. Juan ${ }^{1}$, Horacio María Saint-André2 ${ }^{2}$ Ricardo R. Fernández ${ }^{2}$, \\ Juan Bazzano ${ }^{3}$ y Martín Videla ${ }^{3}$
}

${ }^{1}$ Ing․ Agrónomo, M.Sc., Profesor. Facultad de Agronomía, UNICEN. Casilla de Correo 47. (7300) - Azul, Pcia. de Buenos Aires, Argentina. vjuan@faa.unicen.edu.ar

${ }_{2}^{2}$ Ing ${ }^{\circ}$. Agrónomo, Profesor. Facultad de Agronomia, UNICEN. hsaint@,faa.unicen.edu.ar, rr@faa.unicen.edu.ar

${ }^{3}$ Ing $^{\circ}$. Agrónomo, Ayudante de Investigación. Facultad de Agronomia, UNICEN.

\section{RESUMEN}

Euphorbia dentata es una maleza de difícil control y altamente competitiva en los cultivos de soja en la zona centro de la Provincia de Buenos Aires, Argentina. El desarrollo de soja transgénica resistente a glifosato (RG) permite explorar nuevas técnicas de control químico de malezas, principalmente sobre especies en las cuales los herbicidas tradicionalmente usados no resultan eficientes. El objetivo del presente trabajo fue evaluar la eficacia de diferentes formulaciones de glifosato para el control de E. dentata en soja RG. El ensayo fue realizado en el partido de Azul, Pcia. de Buenos Aires, durante las campañas agrícolas 99/00 y 00/01 utilizando soja CV. AX $4100 \mathrm{RG}$ y con una infestación de E. dentata de $135 \mathrm{pl} / \mathrm{m}^{2}$. Los tratamientos consistieron en la aplicación de tres dosis de glifosato (720, 1260 y $1620 \mathrm{~g} / \mathrm{ha}$ eq.acido) utilizando tres formulaciones comerciales del mismo ingrediente activo existentes en Argentina: formulación 1 (sal isopropylamina SL $48 \%, \mathrm{Ru}$ ), formulación 2 (sal monoamónica WG 74,7\%, Ru_M), formulación 3 (sal isopropylamina tecnología transorb SL $48 \%, \mathrm{Ru}$ _F), testigo limpio y testigo enmalezado. Las aspersiones fueron realizadas cuando la maleza se encontraba en un estado de 4 hojas desarrolladas, con un caudal equivalente a $100 \mathrm{~L} /$ ha, utilizando un equipo manual de $\mathrm{CO}_{2}$. Se evaluó eficacia de control de la maleza y el rendimiento del cultivo. Todas las formulaciones de glifosato lograron altos niveles de control (superiores al 85\%) con la máxima dosis utilizada, no difiriendo significativamente entre sí. La formulación 3 (Ru_F) presentó alta eficacia aún con la dosis de $720 \mathrm{~g}$ /ha donde el resto de la formulaciones logró solo controles parciales (50 y $60 \%$ ). En cuanto al rendimiento, todos los tratamientos difirieron significativamente del testigo enmalezado cuya productividad fue de aproximadamente $2500 \mathrm{~kg} / \mathrm{ha}$. Los tratamientos que lograron los máximos niveles de control no difirieron significativamente del testigo limpio que tuvo un rendimiento de $4000 \mathrm{~kg} / \mathrm{ha}$.

Palabras claves: dosis, formulación.

\section{ABSTRACT \\ Control of Euphorbia dentata with different formulations of glyphosate in transgenic soybean resistant to glyphosate}

Euphorbia dentata is a highly competitive and difficult to control weed in soybean crops in the central area of Buenos Aires Province, Argentina. Development of transgenic soybean resistant to glyphosate (GR) allows exploration of new techniques of chemical weed control, mainly where the herbicides traditionally used are inefficient. The objetive of this research was to evaluate the effectiveness of Euphorbia dentata control with different formulations of glyphosate in soybean GR. The trial was carried out in Azul district, province of Buenos Aires, during the 99/00 and 00/01 agricultural seasons using soybean CV. AX $4100 \mathrm{GR}$ and with an infestation of E. dentata of $135 \mathrm{pl} / \mathrm{m}^{2}$. The treatments consisted in the application 
of three doses of glyphosate (720, 1260 and $1620 \mathrm{~g} /$ ha acid equivalent) using three commercial formulations of the same active ingredient available in Argentina: formulation 1 (isopropylanmine salt SL $48 \%$, Ru), formulation 2 (monoamonic salt WG 74,7\%, Ru_M), formulation 3 (isopropylamine salt with transorb technology SL 48\%, Ru_F), weeded control and non weeded, where the weeds were removed manually. The sprayings were performed when the weed had developed 4 true leaves and with a volume equivalent to $100 \mathrm{~L} / \mathrm{ha}$, using a $\mathrm{CO}_{2}$ manual equipment. Weed control and crop yield were evaluated. All the glyphosate formulations obtained high levels of control (more than $85 \%$ ) with the use of the maximum doses but did not differ significantly among themselves. The isopropylamine salt formulation $(\mathrm{Ru}$ F $)$ showed high efficiency even with a doses of $720 \mathrm{~g} / \mathrm{ha}$, whereas the other formulations only obtained partial controls $(50$ and $60 \%)$. Concerning yield, all the treatments differed significantly from the weeded control whose productivity was about $2500 \mathrm{~kg} / \mathrm{ha}$. The treatments which obtained the maximum levels of control did not differ significantly from the control without weeds which had a yield of 4000 $\mathrm{kg} / \mathrm{ha}$.

Key words: doses, formulation.

\section{INTRODUCCIÓN}

En la República Argentina, la introducción de soja transgénica resistente a glifosato (RG) ha permitido el desarrollo de nuevas técnicas de manejo de malezas, principalmente sobre aquellas especies en las cuales los métodos tradicionalmente utilizados no resultaron efectivos.

Euphorbia dentata es una maleza anual que se encuentra distribuida en el 85\% del área agrícola en la zona centro de la Provincia de Buenos Aires, Argentina, con densidades promedio de 120 plantas $/ \mathrm{m}^{2}$ y con extremos que varían entre 20 a 200 plantas $/ \mathrm{m}^{2}$ (Juan et al., 1996). La misma se destaca por ser altamente competitiva en los cultivos de soja, causando mermas de crecimiento y rendimiento que oscilan entre el 20 y hasta mas del $50 \%$ de acuerdo a las densidades (Juan y Saint-André, 1995; Juan et al., 2000). Por otro lado, estudios tendientes a evaluar el grado de control de la mayoría de los herbicidas y mezclas registradas para soja en Argentina hasta la introducción de los cultivares RG, mostraron la dificultad de control de esta especie por la baja eficacia de los mismos (Juan y Saint-André, 1997 ; Juan et al., 2000).

Por estos motivos y desde el desarrollo de la siembra directa en la zona de estudio, el control químico de $E$. dentata a través del uso de glifosato, como herbicida total aplicado en los barbechos químicos, fue utilizado como una alternativa para disminuir los niveles de infestación de esta especie, pero su éxito estuvo asociado a dosis relativamente altas (aproximadamente $1800 \mathrm{~g} / \mathrm{ha}$ ) y principalmente condicionado por el balance entre las emergencias durante período de barbecho $\mathrm{y}$ las posteriores al nacimiento del cultivo que limitaban el uso de este producto no selectivo por entonces.

Actualmente, y ante la posibilidad de introducir glifosato como herbicida post-emergente en soja RG, se ha replanteado el manejo de muchas especies consideradas como problemáticas a través del uso de este producto en forma selectiva; para ello se han comenzado a realizar ensayos específicos que permitan ajustes de dosis, métodos de aplicación, uso de adjuvantes, etc. Además se registran en el mercado distintas formulaciones del mismo ingrediente activo que han presentado diferencias en cuanto a su eficacia para el control de otras especies.

El objetivo del presente trabajo fue evaluar la eficacia de diferentes formulaciones de glifosato para el control de $E$. dentata en soja RG.

\section{MATERIALES Y MÉTODOS}

Los trabajos fueron llevados a cabo en condiciones de campo, durante las campañas agrícolas 1999/00 y 2000/01, en el partido de Azul, Provincia de Buenos Aires, República Argentina. En lotes seleccionados por su historia de alta infestación de $E$. dentata, fueron implantados cultivos de soja AX 4100 RG (grupo IV) a $35 \mathrm{~cm}$ entre hileras, en parcelas experimentales de $4 \mathrm{~m} \times 10 \mathrm{~m}$. El sitio experimental correspondió a un suelo argiudol típico con $4 \%$ de materia orgánica. Desde la emergencia, las parcelas fueron desmalezadas manualmente eliminando otras especies de malezas, y estableciendo una densidad homogénea de $E$. dentata de 135 plantas $/ \mathrm{m}^{2}$. Cuando el $85 \%$ del stand de esta especie alcanzó el estado de 4 hojas desarrolladas y el cultivo se encontraba en estadío fenológico V3, fueron realizados los tratamientos que consistieron en tres dosis de glifosato $(720,1260$ y $1620 \mathrm{~g} / \mathrm{ha}$ eq. acido) utilizando tres formulaciones comerciales existentes en Argentina: formulación 1 (sal isopropylamina SL $48 \%=\mathrm{Ru}$ ), formulación 2 (sal monoamónica WG $74,7 \%=$ Ru_M), formulación 3 (sal isopropylamina tecnología transorb SL $48 \%=\mathrm{Ru} \_$F), testigo limpio y testigo enmalezado. Las aspersiones se realizaron con equipo manual de presión constante $\left(\mathrm{CO}_{2}\right)$, provisto de una barra aspersora de 4 picos con pastillas 8001 , presión de $2,8 \mathrm{~kg} / \mathrm{cm}^{2}$ y un caudal equivalente a $100 \mathrm{~L} / \mathrm{ha}$. Se realizaron evaluaciones de eficacia de control y fitotoxicidad a los 15 y 35 días desde la aplicación (DDA) y de rendimiento de soja a la cosecha. Se utilizó un diseño de bloques al azar con 4 repeticiones. Los datos se analizaron estadísticamente a través de análisis de varianza y las medias fueron comparadas por el test de Student-Newman-Keuls con $\mathrm{p} \leq 0,05$. 


\section{RESULTADOS Y DISCUSIÓN}

La eficacia de control de $E$. dentata evaluada a los 15 DDA, resultó inferior al 70\%, para todos los tratamientos ensayados excepto para formulación 3 (Ru_F) a razón de 1620 $\mathrm{g} /$ ha que logró, en esta evaluación preliminar, un $75 \%$ de eficacia y difirió significativamente del resto de los productos y dosis (Figura 1).

Luego de 35 días se observó un incremento de los niveles de control obtenidos por todos los tratamientos, destacándose la eficacia de las mayores dosis probadas cuyos controles oscilaron entre el 85 y $93 \%$, no presentando diferencias entre los productos comerciales utilizados.

Niveles de control inferiores se obtuvieron con glifosato a la dosis de $1260 \mathrm{~g} / \mathrm{ha}$ (70-80\%), independientemente del producto comercial utilizado los cuales no presentaron diferencias significativas entre sí. Por otro lado, una baja eficacia fue obtenida con las menores dosis probadas para las formulaciones 1 y 2 (Ru y Ru_M) que no alcanzaron el 60\% de control, no obstante Ru F a razón de 720 g/ha controló un $75 \%$ de las plantas presentes, sin presentar diferencias con respecto a ninguno de los tratamientos en los que se utilizó $1260 \mathrm{~g} / \mathrm{ha}$. En ningún caso fueron observados síntomas de fitotoxicidad debidos a los tratamientos.

Diversos autores han estudiado las diferencias en eficacia de control de las distintas sales de glifosato y destacan la importancia de los adjuvantes que componen las distintas formulaciones comerciales en cuanto a la efectividad obtenida (Wells, 1989; Nalewaja y Matysiak, 1993; Leaper y Holloway, 2000).

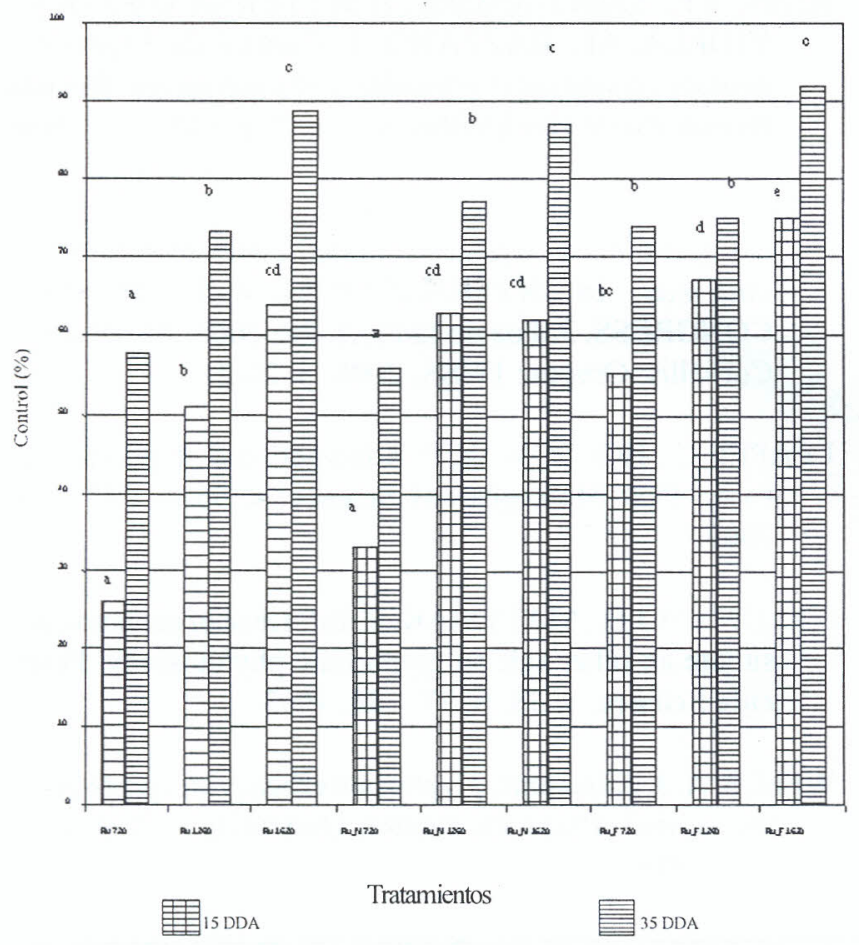

Figura 1. Eficácia de control de E. dentata a los 15 y 35 DDA. Azul, Pcia. de Buenos Aires, 2000.
Feng et al. (1998 y 2000), han determinado diferencias en la eficacia de absorción y transporte en dos formulaciones de glifosato con igual concentración de sal isopropylamina que incluyen distintas mezclas de surfactantes catiónicos, y destacan que estos parámetros podrían explicar los mayores niveles de control que se obtienen entre los productos comerciales sobre todo en malezas de difícil control. En este sentido, este trabajo muestra que la dosis mas baja de $\mathrm{Ru} F$ obtuvo niveles de control significativamente más altos que las otras dos formulaciones probadas, los que pudieron ser detectados desde la primer evaluación realizada a los $15 \mathrm{DDA}$ y que pueden ser consecuencia de una mayor eficiencia de absorción y transporte que en nuestro caso no fue determinada.

La importancia de los altos grados de control obtenidos con glifosato a $1620 \mathrm{~g} / \mathrm{ha}$, se pone de manifiesto cuando se comparan estos resultados con otros datos de control de esta especie realizados en soja no transgénica. Estudios anteriores indican como máximo un $80 \%$ de control de $E$. dentata con imazethapyr en estados fenológicos muy tempranos de la maleza, con poca probabilidad de ocurrencia en la práctica (cotiledón - 2 hojas), y controles que no alcanzan al $70 \%$ cuando la especie tenía 4 hojas desarrolladas con éste mismo herbicida y otras mezclas registradas en Argentina como selectivas en soja no RG (Juan y Saint-André, 1997; Juan et al., 2000).

En cuanto al rendimiento del cultivo, todos los productos y dosis ensayadas difirieron significativamente del testigo enmalezado cuya productividad fue de aproximadamente $2500 \mathrm{~kg} / \mathrm{ha}$ (Figura 2).

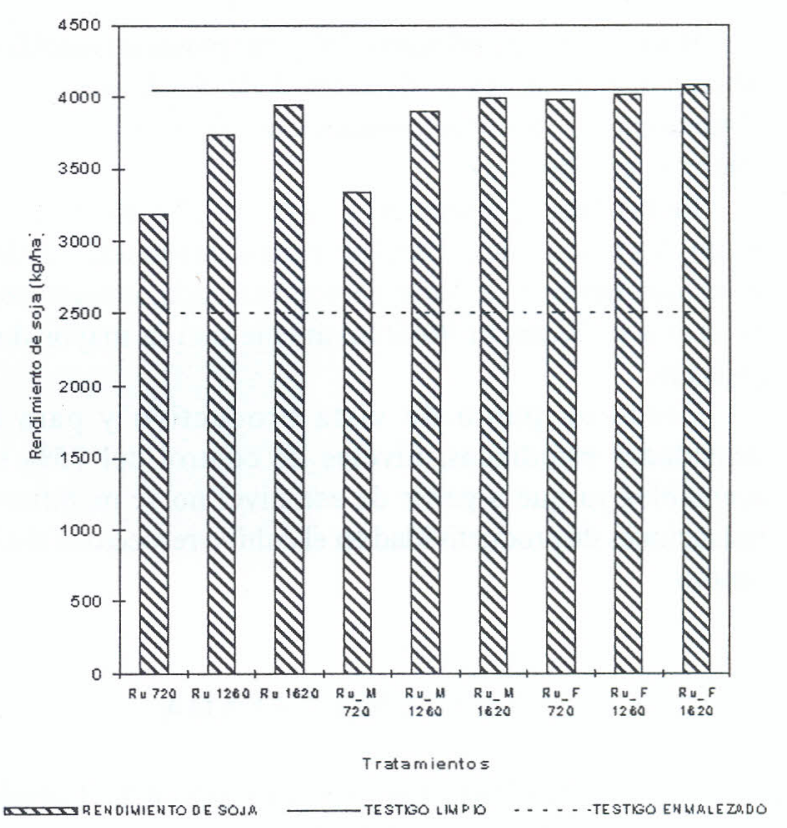

Figura 2. Rendimiento de soja a cosecha. Azul, Pcia. de Buenos Aires, 2000. 
Los tratamientos que no lograron buenos niveles de control de la maleza ( $<60 \%$ de eficacia), sufrieron mermas de rendimiento atribuibles a las densidades remanentes de $E$. dentata resultantes de esos bajos niveles de control y en consecuencia difirieron significativamente del testigo limpio cuya productividad fue de $4000 \mathrm{~kg} / \mathrm{ha}$. Los demás tratamientos, que tuvieron controles cercanos al $75 \%$ ó mayores, no presentaron diferencias de rendimiento con dicho testigo a pesar que la dosis de glifosato de $1620 \mathrm{~g} / \mathrm{ha}$ en los tres productos comerciales utilizados, resultó significativamente superior en cuanto a su eficacia de control (Figura 1).

Por un lado, de acuerdo los resultados obtenidos en este estudio, niveles de control de aproximadamente $75 \%$ serían suficientes para prevenir los efectos de mermas de productividad en soja, provocados por E. dentata a las densidades promedio que se presentan en la zona centro de la Provincia de Buenos Aires. Por otro lado, se sabe que con similares grados de control obtenidos con herbicidas como imazethapyr y algunas mezclas, el área de infestación de esta especie y la densidad promedio se viene incrementando anualmente (Juan et al., 1996).

Lograr niveles de control superiores en soja RG, implica un aumento en la dosis de glifosato de manera tal de obtener una eficacia de alrededor del $90 \%$ que no representará un beneficio adicional para ese cultivo debido a que no se manifestará en una mayor productividad, pero quizás permita sino una reducción, al menos un mantenimiento de las densidades de E. dentata actuales.

\section{CONCLUSIONES}

Bajas dosis de glifosato $(720 \mathrm{~g} / \mathrm{ha})$ ponen de manifiesto diferencias en el grado de control de $E$. dentata que se obtiene con las distintas formulaciones, siendo Ru_F la mas eficiente en este caso.

En las dosis intermedias y altas (1260 y $1620 \mathrm{~g} / \mathrm{ha} \mathrm{eq.}$ acido) los niveles de control obtenidos dependen mas de la dosis que del tipo de formulación utilizada, obteniéndose controles cercanos al $90 \%$ solamente con la mayor dosis probada.

Desde un punto de vista productivo y para las densidades estudiadas, niveles de control del 75\% son aceptables ya que a partir de ese nivel no se manifiestan reducciones de productividad en el cultivo respecto al testigo limpio.

\section{LITERATURA CITADA}

FENG, P.C.C.; RYERSE, J.S.; SAMMONS, R.D. Correlation of leaf damage with uptake and traslocation of glyphosate in velvetleaf (Abutilon theophrasti). Weed Technology, v. 12, p. 300-307, 1998.

FENG, P.C.C.; SANDBRINK, J.J.; SAMMONS, R.D. Retention, uptake and traslocation of ${ }^{14} \mathrm{C}$-glyphosate from track-spray applications and correlation to rainfastness in velvetleaf (Abutilon theophrasti). Weed Technology, v. 14, p. $127-132,2000$.

JUAN, V. F.; SAINT-ANDRE, H. M. Comportamiento de Euphorbia dentata en la zona centro de la Provincia de Buenos Aires: biología de la germinación y sus efectos competitivos sobre el crecimiento de soja. In: CONGRESO LATINOAMERICANO DE MALEZAS, 12. Montevideo, Uruguay, 1995. Actas... Montevideo: INIA, Serie Técnica 56, 1995. p. 174-178.

JUAN, V. F.; SAINT-ANDRE, H. M. Control de lecherón (Euphorbia dentata) en soja con imazethapyr aplicado en distintos estados fenológicos. In: CONGRESO LATINOAMERICANO DE MALEZAS, 13. Buenos Aires, Argentina, 1997. Actas... Buenos Aires: ASAPROVE, 1997, v. 2 p. 128 - 133.

JUAN, V. F.; SAINT-ANDRE, H. ; CARBONE, E.; ORFILA, E.; SCARAMUZZINO, R. L. Estudios sobre lecherón (Euphorbia dentata Michaux) en la zona centro de la provincia de Buenos Aires. Planta Daninha, v. 14, n. 2, p. 102-109, 1996.

JUAN, V.F.; SAINT-ANDRE, H.M.; FERNÁNDEZ, R.R.; VIDELA, M.; BAZZANO, J. Control de Euphorbia dentata en soja con herbicidas post emergentes. Revista Brasileira de Herbicidas, v. 1, n. 2, p. 147-151, 2000.

JUAN, V.F.; SAINT-ANDRE, H.M.; FERNÁNDEZ, R.R. Competition of Euphorbia dentata with soybean (Glycine max). In: INTERNATIONAL WEED SCIENCE CONGRESS, 3. Foz do Iguassu, PR, 2000. Abstracts... Corvallis, Oregon: IWSS, 2000. p. 20-21.

LEAPER C.; HOLLOWAY, P. Adjuvants and glyphosate activity. Pest Management Science, v. 56, p. 313-319, 2000 .

NALEWAJA J.D.; MATYSIAK, R. Influence of diammonium sulfate an other salt on glyphosate phytotoxicity. Pesticide Science, v. 38, p. $77-84,1993$.

WELLS, A. J. Adjuvants, glyphosate efficacy and post-spraying rainfall. Plant Protection Quarterly, v. 4, p. 158163, 1989. 